\title{
Edición universitaria en el contexto de la edición científica: autoría, reconocimiento y valoración
}

\author{
Por José-Antonio Cordón-García y Raquel Gómez-Díaz
}

\begin{abstract}
Resumen: La edición representa un componente sustancial en las actividades de cualquier universidad, por cuanto canaliza en gran parte la investigación que se produce en ella. Se trata de una producción significativa desde los puntos de vista cuantitativo y cualitativo, aunque escasamente considerada en los sistemas de acreditación. Los cambios operados en el sistema de publicación de la ciencia, y los propios de un contexto editorial sometido a profundas transformaciones, obligan a estas entidades a introducir mejoras relacionadas con la función editorial, la elaboración de catálogos y los formatos de publicación.

Palabras clave: Edición científica, Edición universitaria, $V i$ sibilidad, Acreditación, Edición electrónica.

Title: University publishing in the context of scientific publishing: authorship, recognition and evaluation
\end{abstract}

Abstract: Scientific publishing is a substantial component in the activities of any university, channeling much of the research that occurs there. This productivity is significant from both the quantitative and qualitative viewpoints, although scarcely considered in the accreditation systems. The changes in scientific publishing, characteristic of a publishing context that has undergone profound transformations, require these entities to make improvements related to the editorial function, development of catalogs and publishing formats.

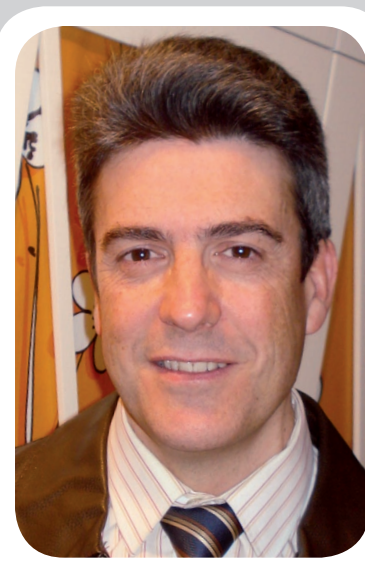

José-Antonio Cordón-García es profesor titular de la Universidad de Salamanca y dirige el Máster de Edición de la Universidad de Salamanca - Grupo Santillana. Es miembro del consejo ejecutivo del Instituto del Libro y la Lectura y presidente de la Asociación Española de Bibliología. Su investigación se centra en la industria editorial y la edición científica, sobre lo que ha publicado monografías y artículos. Dirige la revista Pliegos de Yuste.

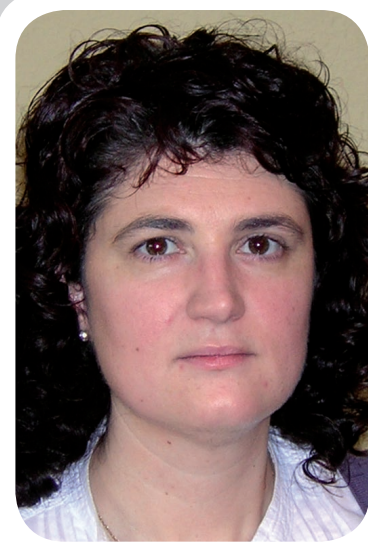

Raquel Gómez-Díaz es doctora en documentación por la Universidad de Salamanca y profesora de dicha universidad. Pertenece al grupo Reina (Recuperación de Información Avanzada) y es miembro del Instituto universitario de estudios de ciencia y tecnología de esa universidad. Cuenta con distintas publicaciones sobre recuperación de la información y sobre documentación electrónica en las que aborda el tema desde diversos puntos de vista.

Keywords: Scientific publishing, Academic publishing, Visibility, Accreditation, Electronic edition.

Cordón-García, José-Antonio; Gómez-Díaz, Raquel. "Edición universitaria en el contexto de la edición científica: autoría, reconocimiento y valoración”. El profesional de la información, 2010, enero-febrero, v. 19, n. 1, pp. 28-34.

DOI: 10.3145/epi.2010.ene.04

\section{Edición universitaria: consideraciones generales}

La mayoría de grandes universidades tiene en la actualidad un servicio de publicación de libros y revistas. Pocas universidades en España se plantean la investigación sin un correlato claro en un dispositivo de transmisión de conocimientos y resultados de investigación que suele revestir la forma de un servicio o secretariado de publicaciones o, directamente, de una editorial universitaria.

Según el Informe Precisa (2008), de las 59 editoriales que analiza, 53 se constituyen como un servicio de publicaciones. De ellas, 44 (el 74,1\%) con sello propio de carácter público. Un $6,8 \%$ son servicio de publica- ciones con sello propio de gestión privada y un $8,3 \%$ servicio de publicaciones pero sin sello propio.

La producción editorial es cuantitativamente importante dentro del sector de la edición científico-técnica. En 2007 las editoriales universitarias españolas publicaron 4.756 títulos, el $7 \%$ de la producción nacional de libros, y cuentan ya con un fondo vivo de 33.496 títulos, el 10\% de los libros en circulación en España (UNE, 2008), aunque la producción global universitaria ha disminuido un $3,6 \%$ con respecto a la de 2005 (Informe Precisa, 2006).

La tirada media de estos libros en 2007 fue de 501 ejemplares, cifra muy baja si la comparamos con las de la edición en general, que en el mismo año era de 
5.070, o la propia de la edición científico-técnica y de ciencias sociales y humanidades (Comercio interior del libro, 2008).

La distribución por temas de los títulos bascula preferentemente hacia las ciencias sociales y las humanidades.

Lo curioso es que, a pesar del aserto anterior que vincula la actividad universitaria con la publicación como un binomio inexorable, y a pesar de que las fe-

\begin{tabular}{|l|r|r|r|r|}
\cline { 2 - 5 } \multicolumn{1}{l|}{} & \multicolumn{1}{c|}{$\mathbf{2 0 0 3}$} & \multicolumn{1}{c|}{$\mathbf{2 0 0 5}$} & $\mathbf{2 0 0 7}$ & \multicolumn{1}{c|}{$\begin{array}{l}\mathbf{2 0 0 7 -} \\
\mathbf{2 0 0 5}\end{array}$} \\
\hline $\begin{array}{l}\text { Títulos } \\
\text { editados }\end{array}$ & 4.300 & 4.936 & 4.756 & -180 \\
$\begin{array}{l}\text { Media títulos } \\
\text { por editorial }\end{array}$ & 91 & 97 & 81 & -16 \\
\hline $\begin{array}{l}\text { Ejemplares } \\
\text { editados }\end{array}$ & 2.226 .458 & 2.736 .711 & 2.384 .639 & -352.072 \\
$\begin{array}{l}\text { Media } \\
\text { ejemplares } \\
\text { por editorial }\end{array}$ & 46.105 & 53.661 & 40.418 & -13.243 \\
\hline $\begin{array}{l}\text { Tirada } \\
\text { media }\end{array}$ & 518 & 554 & 501 & -53 \\
\hline
\end{tabular}

Fuente: Las editoriales universitarias en cifras. Unión de editoriales universitarias, 2008. chas de fundación de muchas de las universidades se remontan a décadas atrás, más del 50\% de los servicios de publicaciones universitarios son posteriores a 1980.

De cualquier modo el conjunto de la comunidad universitaria los considera hoy en día como parte inseparable de la institución. Freeman (1996) resalta el hecho evidente de que más de quinientos años de existencia avalan el papel que las editoriales universitarias han jugado en la difusión de la investigación y en la transmisión de conocimiento. Como es bien sabido, la universidad tiene una misión que se fundamenta en tres principios: la docencia, la investigación y la difusión del conocimiento. Este último, tan importante como los anteriores, es realizado fundamentalmente mediante las editoriales universitarias.

Compelidos a publicar por la inercia consustancial al circuito académico, los miembros de la comunidad universitaria suelen aprovechar la existencia de un dispositivo interno previsto para ello para iniciarse en el circuito de la publicación. Normalmente el rito iniciático se abre con la defensa de la tesis doctoral cuya publicación directa, en formatos variados, o directamente como libro, constituye una práctica habitual. En la me-

\begin{tabular}{|l|r|r|r|r|r|r|r|r|r|}
\cline { 2 - 10 } \multicolumn{1}{c|}{} & \multicolumn{3}{c|}{ Tirada media } & $\begin{array}{c}\text { Índice tirada media } \\
\text { (total = 100) }\end{array}$ & \multicolumn{4}{c|}{ Variación \% } \\
\hline & $\mathbf{2 0 0 6}$ & $\mathbf{2 0 0 7}$ & $\mathbf{2 0 0 8}$ & $\mathbf{2 0 0 6}$ & $\mathbf{2 0 0 7}$ & $\mathbf{2 0 0 8}$ & $\mathbf{2 0 0 6 / 0 6}$ & $\mathbf{2 0 0 7 / 0 6}$ & $\mathbf{2 0 0 8 / 0 6}$ \\
\hline TOTAL & 4.905 & 5.070 & 5.035 & 100,0 & 100,0 & 100,0 & 6,2 & 3,4 & $-0,7$ \\
\hline Científico-técnico y universitario & 2.209 & 2.669 & 2.170 & 45,0 & 52,7 & 43,1 & 14,9 & 20,8 & $-18,7$ \\
\hline Total ciencias sociales y humanidades & 3.145 & 2.317 & 2.681 & 64,1 & 45,7 & 53,2 & 60,3 & $-26,3$ & 15,7 \\
\hline - Ciencias sociales y humanidades & - & 2.191 & 2.240 & - & 43,2 & 44,5 & - & - & 2,2 \\
\hline - Derecho y ciencias económicas & - & 2.338 & 1.822 & - & 46,1 & 36,2 & - & - & $-22,1$ \\
\hline - Religión & - & 2.980 & 6.019 & - & 58,8 & 119,5 & - & - & 101,9 \\
\hline
\end{tabular}

Fuente: Las editoriales universitarias en cifras. Unión de editoriales universitarias, 2008.

\begin{tabular}{|c|c|c|c|c|c|c|c|}
\hline & \multicolumn{2}{|c|}{2003} & \multicolumn{2}{|c|}{2005} & \multicolumn{2}{|c|}{2007} & \multirow{2}{*}{$2007 / 2005$} \\
\hline & Absolutos & $\%$ & Absolutos & $\%$ & Absolutos & $\%$ & \\
\hline Títulos editados & 4.300 & 100,0 & 4.936 & 100,0 & 4.756 & 100,0 & $-3,6$ \\
\hline Ciencias sociales-humanas & 2.700 & 62,8 & 2.571 & 52,1 & 2.648 & 55,7 & 3,0 \\
\hline Ciencias humanas & 1.685 & 39,2 & 1.566 & 31,7 & 1.597 & 33,6 & 2,0 \\
\hline Ciencias sociales & 1.015 & 23,6 & 1.055 & 20,4 & 1.051 & 22,1 & 4,5 \\
\hline Científico-técnico & 1.223 & 28,4 & 1.815 & 36,8 & 1.789 & 37,6 & $-1,4$ \\
\hline Ciencias de la salud & 297 & 6,9 & 260 & 5,3 & 252 & 5,3 & $-3,1$ \\
\hline Ciencias experimentales y tecnología & 926 & 21,5 & 1.555 & 31,5 & 1.537 & 32,3 & $-1,2$ \\
\hline Creación literaria & 155 & 3,6 & 152 & 3,1 & 107 & 2,2 & $-29,6$ \\
\hline Divulgación & 115 & 2,7 & 144 & 2,9 & 152 & 3,2 & 5,3 \\
\hline Otras materias & 107 & 2,5 & 253 & 5,1 & 60 & 1,3 & $-76,3$ \\
\hline
\end{tabular}

Fuente: Las editoriales universitarias en cifras. Unión de editoriales universitarias, 2008. 
dida en la que las universidades forman cada vez más doctores y personal investigador, muchos de los cuales intentan proseguir su carrera académica, se va incrementando la cantera de autores potenciales que buscan la publicación de su primer trabajo, normalmente la tesis doctoral, en la editorial de la universidad.

Los datos recogidos en 2006 con motivo de un informe efectuado para la Junta de Andalucía (Cordón-García; Sánchez-Paso, 2007) son contundentes. Aproximadamente un $40 \%$ de los ISBN concedidos a editoriales universitarias andaluzas en ese año lo ha sido para tesis doctorales. En muchos casos son autores vinculados a las respectivas universidades por diversas fórmulas contractuales. En algunos casos las tesis son publicadas de oficio, en formato electrónico. En otros son los autores los que asumen la iniciativa de sacar a la luz una publicación, interesante desde el punto de vista de la investigación, pero menos relevante desde el ámbito estrictamente comercial.

Este ejemplo pone de manifiesto la singularidad de unos servicios y de unas editoriales cuya lógica no es la de cualquier empresa editorial, y que son considerados de manera natural como la primera opción de publicación por parte del investigador novel.

\section{"En 2007 las editoriales universitarias españolas publicaron 4.756 títulos, el 7\% de la producción nacional de libros"}

Como indica González-Gonzalo (2004) el sistema de promoción académico obliga al profesorado a llevar a cabo una grafomanía militante. De ahí que la mayoría de autores que figuran en catálogos universitarios sean profesores de la propia entidad, al igual que los responsables encargados de valorar la publicación o no de sus trabajos, lo que explica que esta no responda en algunas ocasiones a criterios científicos sino a equilibrios inestables en los que los directores de publicaciones intentan, como sarcásticamente apunta Sánchez-Paso (2002), hacer un catálogo "sin infligir grandes ofensas y sin crearse innecesarios enemigos".

A pesar de la afirmación previa y de los reiterados pronunciamientos en clave negativa que consideran esta endogamia bibliográfica como una rémora inquietante, hay que considerar esta práctica como algo previsible.

En primer lugar, los investigadores llevan a cabo sus trabajos en el seno de una institución que presta sus infraestructuras, equipamientos y recursos económicos (mediante retribuciones salariales) y de personal para que las tareas académicas y de investigación puedan llevarse a efecto. Por lo tanto no es en absoluto descabellado que, con los controles oportunos de consejos asesores, comités de lectura o equivalentes, y previo informe externo, la primera opción de publicación de los resultados de investigación la tenga la propia universidad. Aunque no siempre es así y en muchas ocasiones el investigador recurre a editoriales externas como primera elección.

\section{"De los 60 autores de media que han publicado un libro en las editoriales universitarias, $\mathbf{4 0}$ pertenecen a la propia universidad, 12 a otra y 8 no tienen relación con ellas"}

En la mayoría de situaciones no se trata de casos de "infidelidad" académica, sino de una simple valoración estratégica de la desigual distribución del posicionamiento editorial de las distintas empresas, en el medio del cual la edición universitaria ocupa un lugar desplazado y poco operativo. Puesto que una de las metas de todo autor y, cómo no, de todo investigador es alcanzar la máxima visibilidad, la pretensión prioritaria para cualquiera de ellos será la de colocar el artículo o el libro en el sistema que mejores resultados garantice tanto para la circulación como para la disponibilidad del mismo. En este sentido la empresa privada posee mecanismos de distribución mejor engrasados que la edición universitaria que, aunque han ido mejorando con el tiempo todavía necesita una organización y estructura más aquilatada.

En el trabajo sobre la edición universitaria en Andalucía (Cordón-García; Sánchez-Paso, 2007) se examinó entre otras cuestiones el lugar que ocupan las publicaciones de la universidad en el conjunto de las de un autor, y se halló que de cada 6 obras sólo 1 se publica en la universidad. La pauta de comportamiento de los autores es muy similar en todas las universidades, de tal manera que una vez iniciados en las rutinas de la publicación desde la propia universidad, suele producirse una migración generalizada hacia la edición privada, aprovechando las ventajas concurrenciales y estratégicas que ésta ofrece. De cualquier modo esta pauta se sostiene en el tiempo y es uno de los signos distintivos de este tipo de edición.

En el informe publicado en 2008 para caracterizar la edición universitaria se ha introducido por primera vez la proporción de autores de la propia universidad que integra el catálogo. Según este informe de los 60 
autores de media que han publicado un libro en las editoriales universitarias, 40 pertenecen a la propia universidad, 12 a otra y 8 no tienen relación con ellas (UNE, 2008).

Así pues los docentes utilizan los libros publicados en su institución como elementos consustanciales a su carrera académica. Esta exigencia y necesidad de publicación alimentada por la inercia curricular y los sistemas de valoración científica (Nicholas; Rowland; Jamali, 2006) convierten a los servicios de publicaciones en claves estructurales del campo editorial científico-técnico.

\section{Edición universitaria y acreditación}

El hecho de que las editoriales universitarias, en mayor o menor medida, oficien indirectamente como valedoras de la actividad de investigación, en tanto las obras que publican han estado sometidas a un proceso de control de calidad previo, plantea el problema de la contradicción entre un suministro creciente de manuscritos sometidos a su consideración y una demanda decreciente de textos universitarios. Todo ello en un contexto de crecimiento de las devoluciones - manuscritos no aceptados- y de reducción de las tiradas.

El problema surge cuando se consideran los fines de cualquier investigación y de cualquier investigador, esto es, alcanzar la mayor visibilidad con sus escritos, con objeto de ingresar en los sistemas de valoración científica y conseguir que su trabajo sea convenientemente identificado y comentado (Rowlands; Nicholas; Huntington, 2004; Nicholas; Rowland; Jamali, 2006). Los autores suelen recurrir a los servicios de publicaciones de la universidad como primera instancia de investigación, pero una vez consolidada su carrera académica buscan el concurso de editoriales que les garanticen una mejor distribución y venta de sus obras, mejores condiciones económicas en la percepción de derechos de autor y una mejor valoración académica.

\section{"Los autores buscan editoriales que les garanticen una mejor distribución y venta de sus obras"}

Esta es una de las cuestiones más singulares que afectan al sistema de valoración científica de los investigadores en España. La resolución de 11 de noviembre de 2008 de la presidencia de la Comisión Nacional Evaluadora de la Actividad Investigadora (Cneai) sobre los criterios específicos en cada uno de los campos de evaluación es bastante ilustrativa.
En los campos 1 (matemáticas y física), 2 (química), 3 (biología celular y molecular), 4 (ciencias biomédicas), 5 (ciencias de la naturaleza), 6 (ingeniería y arquitectura), los libros y capítulos de libros (objeto principal de publicación de las editoriales universitarias) constituyen el último ítem evaluable, y en ningún caso se cita entre los imprescindibles para obtener una evaluación positiva. Para estos campos: "en la evaluación de los libros y capítulos de libros, si procede, se tendrán en cuenta el número de citas cuando sea posible, el prestigio internacional de la editorial, los editores, la colección en la que se publica la obra y las reseñas recibidas en las revistas científicas especializadas".

Los términos son tan genéricos que su concreción se hace poco menos que imposible. Aunque se podrían establecer criterios objetivables, la evidencia es que hasta el momento no se han establecido con el mismo nivel de concreción que se ha hecho para las revistas. El apéndice I de la resolución, "Criterios que debe reunir un medio de difusión de la investigación (revista, libro, congreso) para que lo publicado en el mismo sea reconocido como de impacto", podría haber incidido en ello, pero a pesar de un encabezamiento que incluye los libros como medio de difusión de la investigación, comprobamos en el texto que en todo momento se refiere a las revistas, excepto en dos casos en los que se especifican aspectos del proceso editorial que pueden aplicarse a los libros.

\section{"La opción de publicación más inmediata que tiene cualquier investigador es la difusión de su trabajo mediante la editorial de su universidad"}

El problema radica en la falta de método y de indicadores claros para medir el prestigio internacional o nacional de una editorial o de la colección en la que se publica la obra. Existe interesante bibliografía sobre evaluación de colecciones en bibliotecas (Aguado, 2006; Massísimo, 2002), al igual que sobre la valoración de obras de referencia y otros productos editoriales, pero carecemos de indicadores claros y medibles que permitan establecer la calidad, proyección y visibilidad de una editorial. Una aproximación a los mismos con un buen sistema de parámetros valorativos lo han establecido recientemente Giménez Toledo y RománRomán (2009).

En los campos 7 (ciencias sociales, políticas, del comportamiento y de la educación), 8 (ciencias económicas y empresariales), 9 (derecho y jurisprudencia), 10 (historia y expresión artística) y 11 (filosofía, filolo- 
gía y lingüística), se introducen criterios nuevos desde el punto de vista de su valoración como el hecho de que la obra esté incluida en una bibliografía independiente del autor o de su entorno o que sea producto de la reflexión documentada.

Pero donde realmente se pondera y adivina el lugar de la monografía en el contexto de la edición científica es en los considerandos finales. Por una parte se consideran relevantes aquellas monografías que no estén publicadas por la institución a la que pertenezca el investigador, o por empresas editoriales vinculadas con su grupo de investigación, excepto si la editorial acredita un proceso riguroso de selección mediante evaluadores externos a las universidades de la Comunidad Autónoma de que se trate o satisfaga los criterios incluidos en el apéndice I de la resolución. Estos criterios entran en abierta contradicción con la lógica del proceso investigador. Como indicábamos anteriormente, en buena lógica, debería de ser la propia institución quien aprovechara el trabajo de sus investigadores canalizando sus trabajos, difundiéndolos y dotándolos de la visibilidad pertinente. En cierto modo la "externalización" de la investigación debería ser, desde esta visión, una vía subsidiaria. Sin embargo, como demuestran las investigaciones efectuadas al respecto (Cordón-García; Sánchez-Paso, 2007) la realidad es muy diferente. Y parte de la responsabilidad estriba en la escasa consideración que tienen desde el punto de vista de la evaluación los trabajos publicados en la propia institución.

\section{"Los sistemas de control de citas utilizados toman como referencia los artículos y en muy pocos casos las monografías"}

Esta situación ha sido modificada recientemente. La Cneai ha rectificado, mediante resolución publicada en el $B O E$ de 1 de diciembre de 2009, la redacción de los supuestos aplicables a estos campos, suprimiendo la consideración negativa de las monografías publicadas por la propia institución. Presumiblemente haya influido en esta decisión la carta remitida a los responsables de la Cneai por editoriales universitarias, rectores de 31 universidades y personalidades del mundo de la investigación y de la ciencia en febrero de 2009, en la que se subrayaba lo anómalo de la situación y las negativas repercusiones que para la investigación representaba.

Pero la visibilidad en el ámbito académico está estrechamente relacionada con un factor: las citas que el autor o la obra reciben dentro de sus respectivas áreas científicas. El problema es que los sistemas de control de citas utilizados, tanto a escala internacional (Citation index), como nacional (Inrecs, en España) toman como referencia los artículos científicos, y en muy pocos casos las monografías (Giménez-Toledo; RománRomán, 2009). Y en muchas áreas de las humanidades y ciencias sociales las revistas científicas no constituyen el principal canal de comunicación académica (Steele, 2006). La verificación de la excelencia o el reconocimiento mediante los libros es una cuestión casi ignorada en las citadas áreas. A pesar de que numerosas monografías pueden tener un elevado nivel de impacto según los términos del análisis bibliométrico, no son consideradas desde esta perspectiva. Butler y Visser (2006) han demostrado el potencial que representaría extender el análisis de citas a estos otros tipos documentales, a pesar de la inversión de tiempo, esfuerzo y dinero que esto supondría. Otras investigaciones han puesto de manifiesto esta misma idea (Cullars, 1992, Hemlim; Gustafson, 1996; Carr et al., 2006; Nederhof, 2006), esto es, extender los análisis de impacto a otros materiales además de las revistas en aquellas disciplinas en las que el libro representa la vía principal de comunicación. Es paradójico que constituyendo las monografías académicas una de las principales vías de promoción y reconocimiento (Cronin; La Barre, 2004), y casi un prerrequisito para la obtención de determinados puestos académicos, queden fuera de los sistemas de valoración científica, o con un carácter subsidiario.

\section{“La edición electrónica y los repositorios institucionales pueden ser cruciales para la difusión y preservación de la investigación universitaria"}

Otra cuestión es plantear si representan un modelo adecuado para la distribución del conocimiento científico. Se ha hablado de la "fetichización de las monografías" (Inside higher, 2005), en el sentido de la sobrevaloración que revisten en determinadas áreas humanísticas. La realidad es que están surgiendo nuevas formas de edición que pueden solventar la crisis que afecta a las monografías, tanto desde un punto de vista estrictamente editorial como académico. El papel de la edición electrónica y de los repositorios institucionales puede ser crucial como alternativa a la difusión y preservación de la investigación universitaria, siempre y cuando se desenvuelvan en un marco adecuado de gestión de la información generada (Steele, 2006), esto es, cuando, como señalan Regazzi y Caliguiri (2006), 
se mantengan los elementos clave en todo sistema de comunicación científica: verificación, registro, difusión y preservación.

Además de estos aspectos de carácter extrínseco, la edición universitaria padece de la falta de reconocimiento de aquellos que deberían de ser sus receptores naturales: la comunidad universitaria. Su presencia en bibliografías recomendadas y lecturas sugeridas por profesores es insignificante. A título de ejemplo, y como anticipo de una investigación que se está llevando a cabo para analizar la edición científica y su

\begin{tabular}{|c|c|c|}
\hline UNIVERSIDAD & Historia & Filología \\
\hline $\begin{array}{l}\text { Universitat Autònoma de } \\
\text { Barcelona }\end{array}$ & 1 & \\
\hline $\begin{array}{l}\text { Universidad Autónoma de } \\
\text { Madrid }\end{array}$ & 3 & \\
\hline University of Cambridge & 4 & 3 \\
\hline Universidad de Cantabria & 3 & \\
\hline University of Chicago & 1 & \\
\hline Universidad Complutense & 2 & \\
\hline Georgetown University & & 2 \\
\hline Universidad de Granada & & 2 \\
\hline Universidad de Huelva & 2 & \\
\hline Universitat Jaume I & & 1 \\
\hline Universidade Aberta Lisboa & & 1 \\
\hline Universidad de Oviedo & & 1 \\
\hline University of Oxford & 2 & 8 \\
\hline Universidad del País Vasco & 1 & \\
\hline $\begin{array}{l}\text { Universidad Pontificia de } \\
\text { Salamanca }\end{array}$ & & 1 \\
\hline Princeton University & 1 & \\
\hline Université de Provence & 1 & \\
\hline Universidad de Salamanca & 9 & 5 \\
\hline $\begin{array}{l}\text { Universidad de Santiago de } \\
\text { Compostela }\end{array}$ & 1 & 4 \\
\hline University of Texas & 2 & \\
\hline $\begin{array}{l}\text { Universidad Nacional de } \\
\text { Educación a Distancia }\end{array}$ & 3 & 1 \\
\hline Universidad de Valladolid & 5 & \\
\hline Universitat de Barcelona & 1 & \\
\hline Universitat Oberta de Catalunya & & 2 \\
\hline Universitat de València & & 1 \\
\hline Yale University & & 1 \\
\hline
\end{tabular}

Referencias pertenecientes a editoriales universitarias visibilidad en Castilla y León, se analizó la presencia de cualquier tipo de monografía publicada por editoriales universitarias, de cualquier país del mundo, en las bibliografías recomendadas por los profesores de las facultades de Filología y de Historia de la Universidad de Salamanca, dos materias en las que la producción editorial es abundante.

El procedimiento ha sido revisar la bibliografía incluida en las guías académicas e identificar las editoriales universitarias, españolas y extranjeras que figuran en ellas. Hay que tener en cuenta que los datos reflejan bibliografías básicas y que a lo largo del curso los distintos profesores la completan, por lo que los datos habrán de ser tomados con precaución, pero en cualquier caso nos parece interesante señalar que el porcentaje de editoriales universitarias es bastante bajo, un $7 \%$ en ambos casos. En historia se han revisado 82 asignaturas y en filología 61.

Como se ha indicado antes, es preciso completar estos datos, pero son indicativos de la precaria situación de la edición universitaria dentro de las propias instituciones que la promueven. Esa situación no deja de ser paradójica cuando el perfil de los clientes es abrumadoramente universitario, como se reconoce en el informe sobre la edición universitaria de la Unión de editoriales universitarias (UNE, 2008).

\begin{tabular}{|l|r|}
\hline \multicolumn{1}{|c|}{$\begin{array}{c}\text { Distribución en porcentaje } \\
\text { según los clientes finales }\end{array}$} & $\mathbf{1 0 0 , 0}$ \\
\hline Estudiantes & 42,6 \\
\hline Docentes/investigadores & 40,0 \\
\hline Público en general & 16,6 \\
\hline Otros & 0,8 \\
\hline
\end{tabular}

Fuente: Las editoriales universitarias en cifras. Unión de editoriales universitarias, 2008

\section{Conclusiones}

La edición universitaria ha desembocado en un terreno en el que conviven dos lógicas contradictorias pero necesariamente concurrentes: la lógica de la publicación y la de la investigación, provocando las tensiones, desencuentros y malentendidos consiguientes, según pese en la balanza de las decisiones editoriales una u otra. Son editoriales que se encuentran en la difícil situación de continuar con unas formas de edición para las que el mercado es cada vez más restrictivo pero sin una alternativa clara para las mismas, y en las que la aparición de los formatos digitales las aboca a constituirse en plataformas privilegiadas de distribución de contenidos electrónicos de carácter científico. 


\section{Bibliografía}

Aguado, Amelia et al. Desarrollo de colecciones en bibliotecas universitarias. Metodología de evaluación. Proyecto de investigación acreditado y financiado por Universidad Nacional de La Plata, Argentina (H432), Período 2006-2007. 2006 (Unpublished) [Project/Business Plan]

http://eprints.rclis.org/9781/1/Aguado\%2C_A._y_otros_Evaluacion_de_ colecciones_en_bibliotecas_universitarias.pdf

Butler, Linda; Visser, Martijn S. "Extending citation analysis to non-source items". Scientometrics, 2006, v. 66, n. 2, pp. 327-343.

Carr, Les et al. Extending journal-based research impact assessment to book-based disciplines, 2006.

http://eprints.ecs.soton.ac.uk/12725/01/bookcite.Htm

Comercio interior del libro, 2008. Madrid: Federación de Gremios de Editores de España, 2009

Cordón-García, José-Antonio; Sánchez-Paso, José-Antonio. Los servi cios de publicaciones de las universidades andaluzas. Cádiz: Servicio de Publicaciones de la Universidad de Cádiz, 2007. ISBN: 978-84-982-8171-2.

Cullars, John. "Citation characteristics of monographs in the fine arts". Library quarterly, 1992, v. 62 , n. 3, pp. 325-342.

Cronin, Blaise; La Barre, Kathryn. "Mickey Mouse and Milton: book publishing in the humanities". Learned Publishing, 2004, v. 17, n. 2, pp. 85-98.

http://www.ingentaselect.com/ rpsv/catchword/alpsp/09531513/v17n2/s3/p85

Freeman, Lisa. "The university press in the electronic future". En: Peek, Robin P.; Newby, Gregory (eds.). Scholarly publishing: the electronic frontier, Cambridge: The MIT Press, 1996, pp. 147-163. ISBN: 978-0-26266168-3.

Giménez-Toledo, Elea; Román-Román, Adelaida. "Assessment of humanities and social sciences monographs through their publishers: a review and a study towards a model of evaluation". Research Evaluation, 2009, v. 18, n. 3, pp. 201-213.

González-Gonzalo, Eduardo. "La edición del libro universitario en el sistema de comunicación de la ciencia”. En: Cátedra-García, Pedro-Manuel; Páiz-Hernández, María-Isabel; López-Vidriero-Abello, María Luisa (coord.). La memoria de los libros: estudios sobre la historia del escrito y de la lectura en Europa y América. Madrid: Instituto de Historia del Libro y de la Lectura, 2004, v. 2, pp. 701-717. ISBN 84-933504-4-3.

Hemlin, Sven; Gustafson, M. "Research production in the arts and humanities: a questionnaire study of factors influencing research performance". Scientometrics, 1996, v. 37, n. 3, pp. 417-432.
Informe Precisa. Comercio interior del libro 2005: editoriales universitarias. Madrid: Asociación de Editoriales Universitarias, 2006.

Inside higher ed. "Radical change for tenure", 30 dec. 2005. http://www.insidehighered.com/news/2005/12/30/tenure

Massísimo-Sánchez-Boado, Àngels. "Evaluación de colecciones en las bibliotecas universitarias (I). Métodos basados en el estudio de la colección". Anales de documentación, 2002, v. 5, pp. 245-272.

http://revistas.um.es/analesdoc/article/viewFile/2111/2101

Nederhof, Anton J. "Bibliometric monitoring of research performance in the social sciences and the humanities: a review". Scientometrics, 2006, v. 66, n. 1 , pp. $81-100$.

Nicholas, David; Rowland, Ian; Jamali, Hamid. "On the tips on their tongues: authors and their views on scholarly publishing". Learning publishing, 2006, v. 19, n.3, pp. 193-203.

Regazzi, John J.; Caliguiri, Nicole A. "Publisher and autor partnerships: a changing landscape". Learned publishing, 2006, v. 19, n. 3, pp. 183-192.

Rowlands, Ian; Nicholas, Dave; Huntington, Paul. Scholarly communication in the digital environment: what do authors want? London: Centre for Information Behaviour and the Evaluation of Research, Dept. of Information Science, City University, pp. 10-12.

Sánchez-Paso, José-Antonio. "Cómo montar una editorial universitaria". Archipiélago: cuadernos de crítica de la cultura, 2002, n. 51, pp. 45-50.

Steele, Colin. "Open all hours? Institutional models for open access". In: Jacobs, N. (ed.), Open access: key strategic, technical and economic aspects. Oxford: Chandos, 2006

http://cogprints.org/4841

Unión de editoriales universitarias españolas. Las editoriales universitarias en cifras: estudio comercio interior 2007 editoriales universitarias. 2008. http://www.une.es/media/Oul/File/Informes/Informe\%20edici\%C3\%B3n\% 20universitaria\%202007_V1.pdf

José-Antonio Cordón-García, Raquel Gómez-Díaz. Facultad de Traducción y Documentación, Universidad de Salamanca.

Francisco Vitoria 6-16, 37004 Salamanca

jcordon@usal.es

rgomez@usal.es

El profesional de la información está indizada por la base de datos

ISI Social Science Citation Index, de Thomson Reuters, y según el Journal Citation Report ha obtenido un Factor de impacto de 0,400 